\title{
ORIGINAL ARTICLE \\ Diagnostic accuracy of diffusion tensor imaging for pediatric cervical spinal cord injury
}

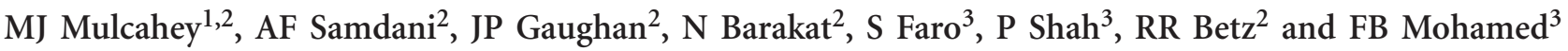

\begin{abstract}
Study design: Cross-sectional non-experimental study.
Objectives: To examine diagnostic accuracy of diffusion tensor imaging (DTI) for pediatric spinal cord injury (SCI).

Setting Pediatric Orthopedic Hospital.

Methods: Thirty-five subjects, $10 \mathrm{SCl}$ and 25 controls, mean age 13.38 years underwent two scans with 3.0T MR scanner. Fractional anisotropy (FA), axial diffusivity (AD) and radial diffusivity (RD) values were calculated. Subjects with $\mathrm{SCl}$ underwent examination of muscle strength, sensation and sacral sparing. Mean and s.d. values for FA, AD and RD were compared by group (controls, $\mathrm{SCl}$ with sacral sparing, $\mathrm{SCl}$ without sacral sparing) using analysis of variance for repeated measures. Comparisons were also made of DTI values at the injury site to values from cervical regions outside of the injury site. Specificity, sensitivity, receiver operating characteristics area under the curve (ROC AUC) and corresponding 95\% confidence intervals were calculated. Resampling methods were used to validate the estimates from the final models.
\end{abstract}

Results: FA values differed among $\mathrm{SCl}$ subjects with intact sacral sparing, absent sacral sparing and controls, $P<0.003$ (adjusted). DTI values in combination showed the strongest diagnostic accuracy for predicting the presence of anal contraction (AD, RD; ROC AUC $=0.90$ ), deep anal pressure $(F A$; ROC $A \cup C=0.88)$, S4-5 sensation (FA, RD; ROC AUC =0.93), motor level $(F A, A D, R D$; ROC AUC $=0.92)$ and MRI level $(F A, A D, R D ; R O C A U C=0.92)$. Bootstrap and Jackknife median values indicated consistency of the parameter estimates.

Conclusion: The predictive accuracy of DTI for sacral sparing end points and motor and MRI level of injury was good to strong. Spinal Cord (2013) 51, 532-537; doi:10.1038/sc.2013.36; published online 23 April 2013

Keywords: tetraplegia; neuroimaging; sensitivity; specificity

\section{INTRODUCTION}

Evidence is mounting in support of diffusion tensor imaging (DTI) as a quantitative method to evaluate spinal cord damage. ${ }^{1-6}$ Several studies have shown that DTI indices such as fractional anisotropy (FA), axial diffusivity ( $\mathrm{AD})$ and radial diffusivity (RD) have the potential to quantify biologically relevant diffusion changes in the spinal cord. $\mathrm{RD}$ and $\mathrm{AD}$ are the rate of diffusion perpendicular and parallel to the white matter tract, respectively. FA represents the degree to which diffusion is anisotropic. High FA values indicate that diffusion is greater in one direction than in other directions, whereas low FA values indicate diffusion is relatively equal in all the directions.

There is a misconception that the neurological status of children with spinal cord injury (SCI) by clinical examination is fairly clear. Although motor level in children $>6$ years of age may be reliable by clinical examination, results of the neurological examination for sensory level and injury completeness in children $<8$ years of age is not reliable. ${ }^{7}$ Agreement about neurological status of children with SCI in medical records is poor ${ }^{8}$ and agreement on repeated sensory and motor scores at individual dermatomes and myotomes in children is only moderate. 9,10

Although most of the DTI research has been with adults, our research center has begun to establish utility for the pediatric spinal cord. ${ }^{11-13}$ We have reported that DTI indices of the cervical spinal cord can be obtained in children with SCIs with moderate-to-strong reliability and that the indices had moderate-to-good concurrent validity against MRI and the International Standards of Neurological Classification of Spinal Cord Injury motor, sensory and anorectal examinations. ${ }^{11}$ The purpose of this study was to evaluate the predictive validity of DTI by examining its diagnostic accuracy for pediatric cervical SCI.

\section{MATERIALS AND METHODS}

The sample consisted of 35 subjects, 10 with SCI and 25 controls (5 male, 20 female). The mean age was $13.4( \pm 3.9)$ and $13.5( \pm 4.6)$ years for the SCI and control groups, respectively. Table 1 summarizes pertinent characteristics of the study sample with SCI.

Youths with SCI had chronic cervical SCI, were independent from mechanical ventilation and did not have cervical spine instrumentation or bullet fragments in the cervical canal. The controls were typically developing without health concerns. Youths were excluded if they had teeth braces or were unable to tolerate imaging without sedation. We certify that all applicable institutional and governmental regulations concerning the ethical use of children as research volunteers were followed during the course of the study.

${ }^{1}$ Occupational Therapy, Thomas Jefferson University, Jefferson School of Health Professions, Philadelphia, PA, USA; ${ }^{2}$ Shriners Hospitals for Children Philadelphia, Philadelphia PA, USA and ${ }^{3}$ Department of Radiology, Temple University, Philadelphia, PA, USA

Correspondence: Dr MJ Mulcahey, Occupational Therapy, Thomas Jefferson University, Jefferson School of Health Professions, 901 Walnut Street, 6th Floor, Philadelphia, PA 19107, USA.

E-mail: maryjane.mulcahey@jefferson.edu

Received 26 October 2012; revised 25 March 2013; accepted 26 March 2013; published online 23 April 2013 
Table 1 Characteristics of the subjects with SCI

\begin{tabular}{|c|c|c|c|c|c|c|c|c|c|c|c|c|}
\hline & $\begin{array}{l}\text { Age at scan } \\
\text { (years) }\end{array}$ & Gender & $\begin{array}{l}\text { Age at injury } \\
\text { (years) }\end{array}$ & Etiology & \multicolumn{7}{|c|}{$\begin{array}{l}\text { International Standards for Neurological Classification of } \\
\text { Spinal Cord Injury clinical end points }\end{array}$} & MRI level \\
\hline SCl_02 & 13 & $\mathrm{~F}$ & 12 & $\mathrm{TM}$ & $\mathrm{C} 6$ & $\mathrm{C7}$ & $\mathrm{c} 6$ & $C$ & $\mathrm{~N}$ & Y & $N$ & $\mathrm{C} 4$ \\
\hline SCl_03 & 15 & $\mathrm{~F}$ & 4 & MVC & $\mathrm{C7}$ & $\mathrm{C} 8$ & $\mathrm{C7}$ & B & $\mathrm{N}$ & Y & $N$ & C5-6 \\
\hline SCl-04 & 11 & M & 0.04 & $\mathrm{TM}$ & $\mathrm{C} 8$ & $\mathrm{~T} 1$ & $\mathrm{C} 8$ & C & Y & Y & Y & C3 \\
\hline SCl-11 & 11 & $\mathrm{~F}$ & 1 & MVC & $\mathrm{C} 5$ & $\mathrm{C} 6$ & $\mathrm{C} 5$ & $A$ & $\mathrm{~N}$ & $\mathrm{~N}$ & $\mathrm{~N}$ & $\mathrm{C} 5$ \\
\hline SCl_12 & 14 & $M$ & 12 & Fall & $\mathrm{C} 1$ & $\mathrm{C} 1$ & $\mathrm{C} 1$ & B & $\mathrm{N}$ & Y & Y & $\mathrm{C} 1$ \\
\hline SCl_13 & 10 & M & 1 & Fall & $\mathrm{C} 1$ & $\mathrm{C} 1$ & $\mathrm{C} 1$ & D & $Y$ & Y & Y & Neg \\
\hline SCl_14 & 17 & M & 13 & Diving & $\mathrm{C} 5$ & $\mathrm{C7}$ & $\mathrm{C} 5$ & B & $\mathrm{N}$ & Y & Y & $\mathrm{C} 4$ \\
\hline SCl_15 & 8 & $\mathrm{~F}$ & 0.06 & MVC & $\mathrm{C8}$ & $\mathrm{C} 8$ & $\mathrm{C} 8$ & B & $\mathrm{N}$ & Y & $\mathrm{N}$ & $\mathrm{C} 4$ \\
\hline SCl_16 & 20 & $\mathrm{~F}$ & 16 & Vascular & $\mathrm{C} 1$ & $\mathrm{C} 1$ & $\mathrm{C} 1$ & D & $Y$ & Y & Y & $\mathrm{C} 2$ \\
\hline
\end{tabular}

Abbreviations: AC, anal contraction; AIS, American Spinal Injury Association Impairment Scale; AIS A, complete injury; AIS B, sensory incomplete injury; AIS C, gravity eliminated motor incomplete injury; AIS D, against gravity motor incomplete injury; DAP, deep anal pressure; F, female; M, male; ML, motor level; MVC, motor vehicle crash; N, no; Neg, negative; NL, neurological level; SL, sensory level; TM, transverse myelitis; Y, yes.

\section{Neurological evaluation}

Subjects with SCI underwent a neurological evaluation based on The International Standards for Neurological Classification of Spinal Cord Injury. ${ }^{14}$ Sensory appreciation to sharp/dull discrimination and light touch was evaluated in 56 dermatomes, including S4-5. Deep anal pressure and voluntary anal contraction were evaluated by digital examination.

\section{Imaging protocol}

Subjects underwent two identical scans using a $3.0 \mathrm{~T}$ Siemens Verio MR scanner (Siemens, Erlangen, Germany) with a 4-channel neck matrix and an 8channel spine matrix coil; the average time between the two scans was $9 \mathrm{~h}$. The protocol consisted of conventional sagittal fast Spin Echo T1- and T2-weighted scans, axial fast Spin Echo T2-weighted scans as well as axial DTI scans using the inner-field-of-view (FoV) pulse sequence with spatially $2 \mathrm{D}$-selective radio frequency excitations. Anesthesia was not administered nor was cardiac and/or respiratory gating employed.

The inner-FoV sequence used in this study has been described in detail elsewhere ${ }^{15}$ and was based on a single-shot echo planar imaging sequence for diffusion-weighted imaging with spatially 2D-selective radio frequency excitations. Although it is common in diffusion-weighted preparations to use two refocusing radio frequency pulses and four gradient pulses, we applied a single refocusing radio frequency pulse with three gradient pulses. The axial DTI images were acquired in the same anatomical location prescribed for the axial fast Spin Echo T2-weighted images to cover the entire cervical spinal cord (C1-T1). The imaging parameters included were: 20 diffusion directions, $\mathrm{b}=1000 \mathrm{~s} \mathrm{~mm}^{-2}$, voxel size $=1.2 \times 1.2 \times 3 \mathrm{~mm}^{3}$, axial slices $=$ $35-45$ (depending on the subject's height), gap $=0 \mathrm{~mm}$, in tegrated parallel acquisition techniques $(\mathrm{iPAT})=0, \quad \mathrm{FoV}=250 \times 43 \mathrm{~mm}$, repetition time $=6100-8000 \mathrm{~ms}$, echo time $=115 \mathrm{~ms}$, number of averages $=3$, and acquisition time $=6-7 \mathrm{~min}$. This imaging protocol was used after optimization of various DTI parameters indicated the highest signal-to-noise ratio achieved within the shortest acquisition time. ${ }^{13}$

\section{Data post-processing}

The diffusion data sets were corrected for motion using the Automated-ImageRegistration package implemented in DTIstudio (www.mristudio.org) (Figure 1). The target images (20 directional images) were aligned with the reference image (b0) using a rigid registration algorithm and scaled-leastsquares cost function. ${ }^{13}$ The eigenvectors and eigenvalues of the diffusion tensor matrix were computed on a voxel-by-voxel basis from the axial DTI images. The region of interest (ROI) analysis method has been described in detail $^{13}$ and is summarized here. ROIs were manually drawn on color FA maps

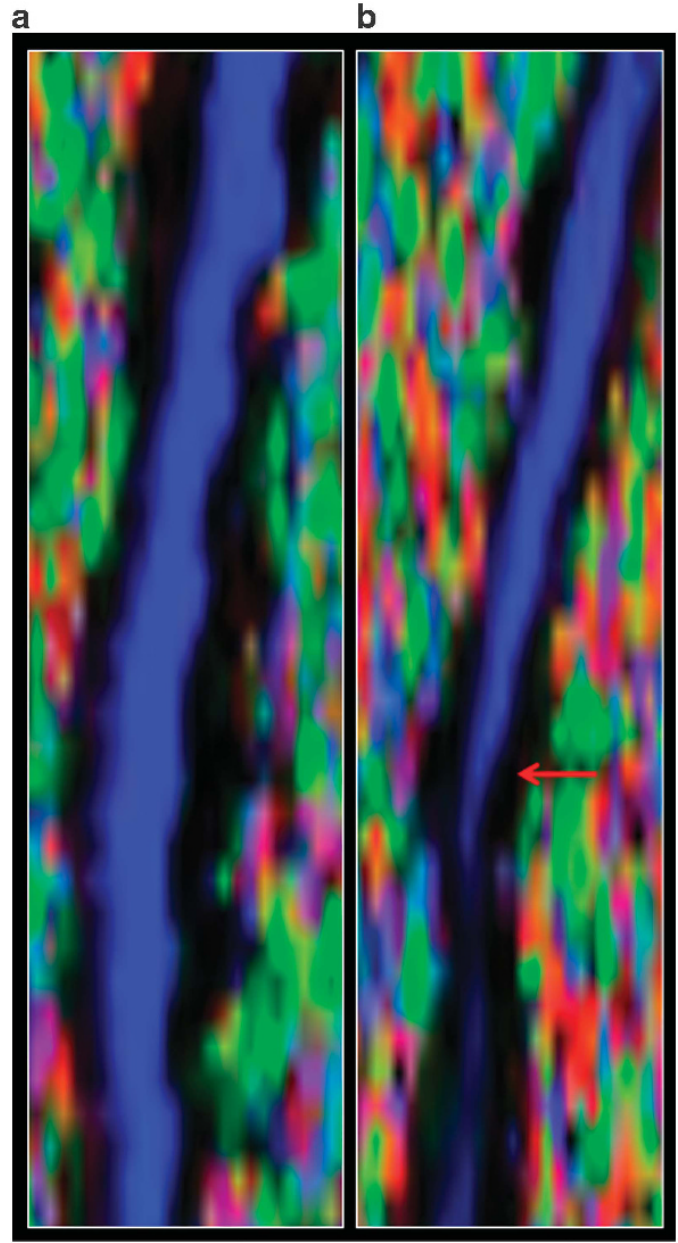

Figure 1 Midline reconstructed sagittal FA color map of a control subject (a) and a subject with $\mathrm{SCl}$ (b). Arrow represents level of injury at $\mathrm{C} 5$.

at every axial slice location along the cervical spinal cord for both acquisitions and were validated by a neuroradiologist to ensure proper anatomical localization. There was a consistent sparring of the outer margin of the 
cervical cord that represented approximately one voxel width to minimize volume averaging with the cerebrospinal fluid. ROIs were consistently defined throughout all slices. $\mathrm{FA}, \mathrm{AD}$ and $\mathrm{RD}$ values were averaged per disk level of the cervical cord as well as the middle portion of the cervical vertebra.

\section{MRI findings}

MRI findings for each level of the cord were classified as 'normal' or 'abnormal' by a board-certified neuroradiologist. Findings were considered abnormal if there was an increased intramedullary signal on the fast Spin Echo T2-weighted images with or without associated cord atrophy. The MRI level was identified by locating the transition point from normal to abnormal.

\section{Statistical analysis}

Mean \pm s.d. values for $\mathrm{FA}, \mathrm{AD}$ and $\mathrm{RD}$ were calculated by level of the cord based on the measurements from both the scans of each subject. Mean values were compared by group (controls, SCI with intact clinical end point, SCI with absent clinical end point) and by region of the cervical cord (motor level, MRI level and cervical region above and below motor and MRI level of injury) using analysis of variance for repeated measures. The clinical end points used to group subjects with SCI were anal contraction, deep anal pressure and S4-5 sensation.

Single and multiple variable logistic regressions were used to analyze DTI parameters as predictors of deep anal pressure, voluntary anal contraction, S4-5 sensation and motor level and MRI level of injury. For univariate analysis, each DTI index was examined as predictor of clinical end points. The multiple variable logistic regression used a combination of DTI indices that showed to be the strongest predictors of clinical end points; these combinations differed depending on the clinical end point. We did not use any other variable (age, gender, cause of injury, and so on) as predictors, only DTI values were used.
Sensitivities and specificities were calculated based on the best combination cut-point chosen from the resulting receiver operating characteristic (ROC). Specificity, sensitivity, ROCs area under the curve (ROC AUC) and corresponding 95\% confidence intervals (CIs) were calculated for each of the resulting models. Jackknife (multiple samples of 1000 with replacement) and bootstrap (one subject out, $n=35$ ) resampling methods were used to validate the estimates from the final models.

\section{RESULTS}

The controls showed an average $\mathrm{FA}=0.54 \pm 0.11, \mathrm{AD}=1.00 \times 10^{-3}$ $\mathrm{mm}^{2} \mathrm{~s}^{-1} \pm 0.18 \times 10^{-3}$ and $\mathrm{RD}=0.39 \times 10^{-3} \mathrm{~mm}^{2} \mathrm{~s}^{-1} \pm 0.12 \times$ $10^{-3}$. The subjects with SCI showed reduced FA and increased diffusivity values compared with the controls: $\mathrm{FA}=0.28 \pm 0.10$, $\mathrm{AD}=1.15 \times 10^{-3} \mathrm{~mm}^{2} \mathrm{~s}^{-1} \pm 0.28 \times 10^{-3}, \quad$ and $\quad \mathrm{RD}=0.80 \times$ $10^{-3} \mathrm{~mm}^{2} \mathrm{~s}^{-1} \pm 0.27 \times 10^{-3}$.

There were significant differences in mean FA values between the control group, the SCI group with intact sacral sparing and the SCI group with absent sacral sparing (Table 2, Figures $2 \mathrm{a}-\mathrm{c}$ ). AD values were significantly different among the control group, SCI group with anal contraction and the SCI group without anal contraction. However, for the remaining comparisons of both $\mathrm{AD}$ and $\mathrm{RD}$, differences were significant only between the control group and SCI group with absent deep anal pressure and S4-5 sensation. Table 2 summarizes DTI parameters at the MRI and motor level of injury compared with cervical levels above and below the injury level.

There was a strong association between FA, AD and RD and anal contraction, deep anal pressure, S4-5 sensation, MRI findings and severity of injury. Univariate analysis indicated that FA was the

Table 2 Mean and s.d. values for fractional anisotropy (FA); axial diffusivity (AD); radial diffusivity (RD) for controls, SCI groups with and without AC, DAP and S4-5 sensation; controls, complete and incomplete injuries and; for motor level as defined by ISNCSCI and for MRI level for the control group, $\mathrm{SCl}$ group at the level of injury and $\mathrm{SCl}$ group at cervical regions above and below injury level

\begin{tabular}{|c|c|c|c|c|c|c|}
\hline & \multicolumn{2}{|c|}{$F A$} & \multicolumn{2}{|c|}{$A D$} & \multicolumn{2}{|c|}{$R D$} \\
\hline & Mean & s.d. & Mean & s.d. & Mean & s.d. \\
\hline \multicolumn{7}{|l|}{ Anal contraction $(A C)$} \\
\hline $\mathrm{SCl}$ absent & $0.28^{a}$ & 0.13 & $1.23^{\mathrm{a}}$ & 0.44 & $0.87^{a}$ & 0.47 \\
\hline $\mathrm{SCl}$ intact & $0.34^{\mathrm{a}}$ & 0.05 & $0.71^{\mathrm{a}}$ & 0.29 & 0.43 & 0.18 \\
\hline $\mathrm{SCl}$ absent & $0.24^{a}$ & 0.12 & $1.28^{\mathrm{a}}$ & 0.68 & $0.99^{a}$ & 0.68 \\
\hline $\mathrm{SCl}$ intact & $0.31^{\mathrm{a}}$ & 0.11 & 1.01 & 0.37 & 0.67 & 0.35 \\
\hline Control & $0.51^{\mathrm{a}}$ & 0.08 & 0.98 & 0.27 & 0.41 & 0.11 \\
\hline \multicolumn{7}{|l|}{$s 4-5$} \\
\hline $\mathrm{SCl}$ absent & $0.26^{a}$ & 0.14 & $1.30^{\mathrm{a}}$ & 0.44 & $0.94^{a}$ & 0.48 \\
\hline $\mathrm{SCl}$ intact & $0.35^{\mathrm{a}}$ & 0.05 & 0.75 & 0.26 & 0.45 & 0.16 \\
\hline \multicolumn{7}{|l|}{ Region of cervical spinal cord } \\
\hline \multicolumn{7}{|l|}{ Motor level } \\
\hline SCI—ISNCSCI motor level of injury & 0.28 & 0.13 & $0.81^{\mathrm{a}}$ & 0.43 & $0.6^{\mathrm{a}}$ & 0.43 \\
\hline $\mathrm{SCl}$-cervical regions above and below ISNCSCI mtor level of injury & 0.31 & 0.11 & $1.2^{\mathrm{a}}$ & 0.43 & $0.8^{\mathrm{a}}$ & 0.45 \\
\hline Control & $0.51^{\mathrm{a}}$ & 0.08 & $0.98^{\mathrm{a}}$ & 0.27 & $0.41^{\mathrm{a}}$ & 0.12 \\
\hline \multicolumn{7}{|l|}{ MRI level } \\
\hline SCl—MRI level of injury & $0.23^{a}$ & 0.13 & $1.26^{\mathrm{a}}$ & 0.49 & $0.96^{a}$ & 0.52 \\
\hline $\mathrm{SCl} —$ cervical regions above and below MRI level of injury & $0.35^{\mathrm{a}}$ & 0.08 & 0.92 & 0.38 & $0.55^{\mathrm{a}}$ & 0.27 \\
\hline Control & $0.51^{\mathrm{a}}$ & 0.08 & 0.98 & 0.27 & $0.41^{\mathrm{a}}$ & 0.12 \\
\hline
\end{tabular}

Abbreviations: ISNCSCI, International Standards for Neurological Classification of Spinal Cord Injury; MRI, magnetic resonance imaging.

andicates significant difference, $P<0.003$ (adjusted). 
a

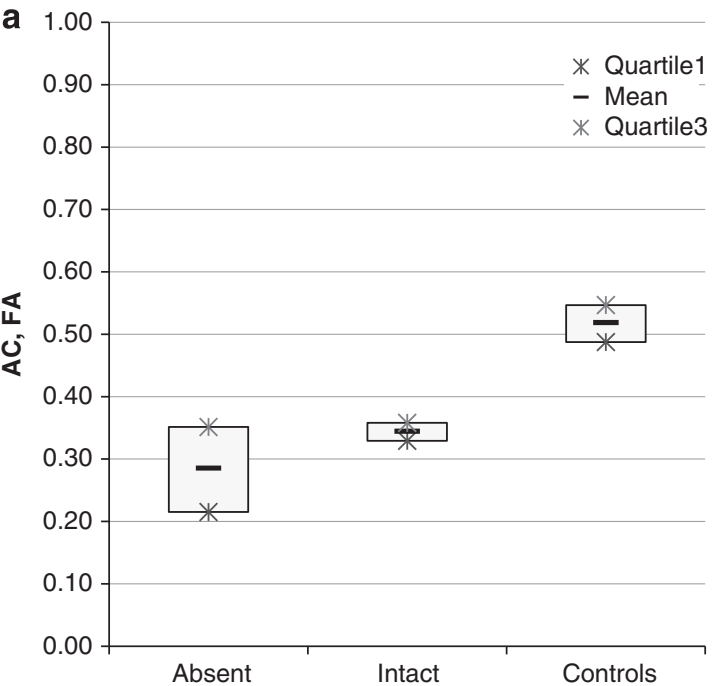

b

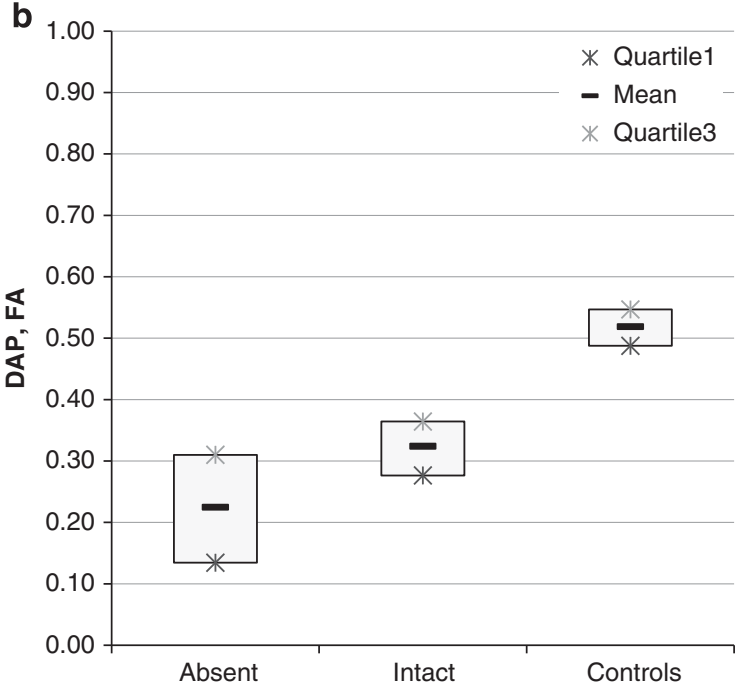

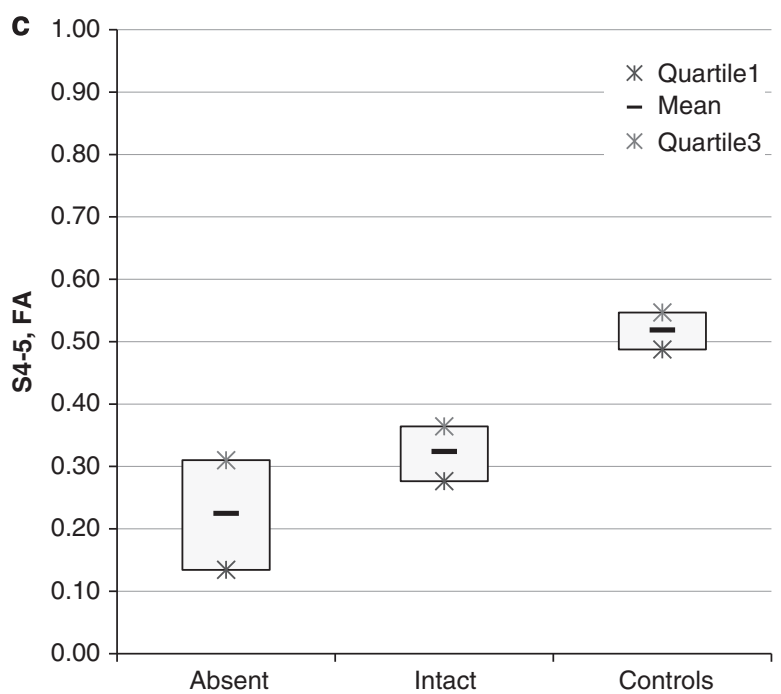

Figure 2 (a) Trends in fractional anisotropy for subjects with $\mathrm{SCl}$ with absent anal contraction, intact anal contraction and in controls. $Y$ axis indicates range of FA values from low (abnormal) to high (normal); $x$ axis $=\mathrm{SCl}$ subjects with absent (AC); $\mathrm{SCl}$ subjects with intact $(\mathrm{AC})$ and controls. (b) Trends in fractional anisotropy for subjects with $\mathrm{SCl}$ with absent deep anal pressure, intact deep anal pressure and in controls. DAP $=$ deep anal pressure; $y$ axis indicates range of FA values from low (abnormal) to high (normal); $x$ axis $=\mathrm{SCl}$ subjects with absent (DAP); SCl subjects with intact (DAP) and controls. (c) Trends in fractional anisotropy for subjects with SCl with absent S4-5 sensation, intact S4-5 sensation and in controls. S4-5=sacral dermatome corresponding with sacral level 4-5; $y$ axis indicates range of FA values from low (abnormal) to high (normal); $x$ axis $=\mathrm{SCl}$ subjects with absent (S4-5 sensation); $\mathrm{SCl}$ subjects with intact ( $\mathrm{S} 4-5$ sensation) and controls.

Table 3 Multivariate analysis showing specificity, sensitivity and receiver operating characteristic area under the curve (ROC AUC) and 95\% confidence intervals (Cls) of DTI values for AC, DAP, S4-5 sensation, motor level and MRI level

\begin{tabular}{lllll}
\hline & Predictor & Specificity $(95 \%$ CI) & Sensitivity $(95 \%$ CI) & ROC AUC (95\% CI) \\
\hline Anal contraction (AC) & AD, RD & $0.74(0.71,0.77)$ & $0.80(0.75,0.84)$ & $0.93(0.91,0.94)$ \\
Deep anal pressure (DAP) & FA & $0.53(0.50,0.56)$ & $0.70(0.60,0.79)$ & $0.88(0.85,0.90)$ \\
S4-5 & FA, RD & $0.81(0.78,0.83)$ & $0.80(0.75,0.85)$ & $0.93(0.92,0.95)$ \\
Motor Level & FA, AD, RD & $0.67(0.65,0.70)$ & $0.85(0.78,0.90)$ & $0.92(0.91,0.94)$ \\
MRI Level & FA, AD, RD & $0.83(0.80,0.85)$ & $0.89(0,84,0.93)$ & $0.92(0.90,0.94)$
\end{tabular}

Abbreviations: AD, axial diffusivity; DTI, diffusion tensor imaging; FA, Fractional anisotropy; MRI, magnetic resonance imaging; RD, radial diffusivity.

strongest overall predictor of each clinical end point. However, DTI values in combination showed the strongest diagnostic accuracy for predicting the presence of anal contraction ( $\mathrm{AD}$ and $\mathrm{RD})$, deep anal pressure (FA), S4-5 sensation (FA, RD), motor level (FA, AD and RD) and MRI level (FA, AD and RD) (Table 3, Figures 3 and 4). Bootstrap and Jackknife median values from the re-sampling procedures were similar to the values from the final model, indicating consistency of the parameter estimates. 


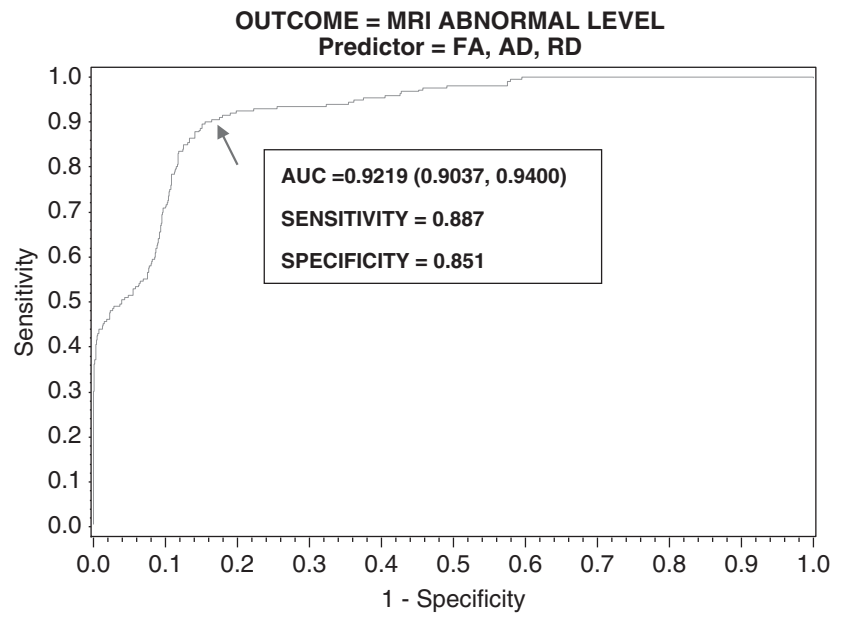

Figure 3 ROC curve shows the relationship between sensitivity (true positives; $y$ axis) and 1 -specificity (false positives; $x$ axis) of DTI for MRI abnormal level. $A U C=0.92$, indicating $92 \%$ accuracy of DTI parameters predicting abnormal MRI level.

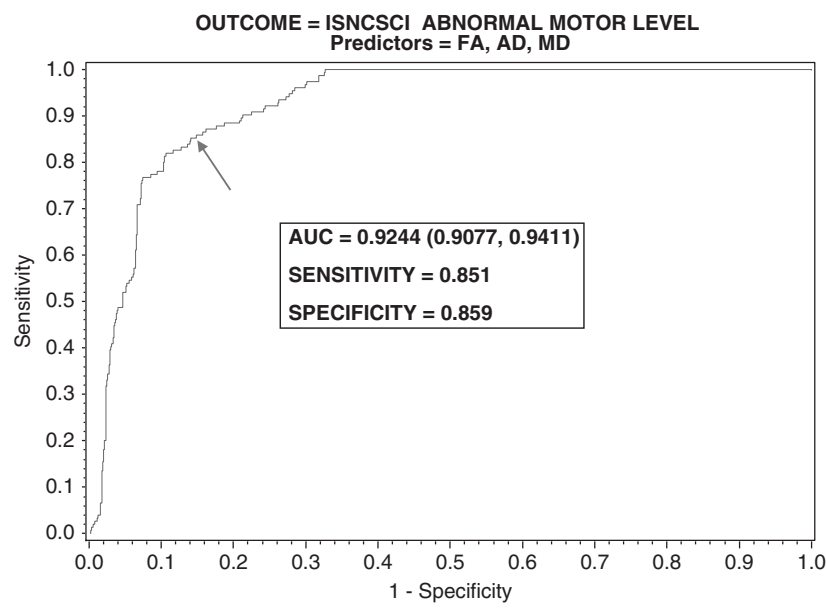

Figure 4 ROC curve shows the relationship between sensitivity (true positives; $y$ axis) and 1 -specificity (false positives; $x$ axis) of DTI for INSCSCI abnormal motor level. AUC $=0.92$, indicating $92 \%$ accuracy of DTI parameters predicting abnormal MRI level.

\section{DISCUSSION}

When compared with children without SCI, the subjects with SCI showed reduced FA values and increased diffusivity values. This finding is consistent with adult reports ${ }^{2,4,6}$ and provides additional impetus to further develop DTI as an objective method to evaluate the consequence of SCI for adults and children. Although previous studies have compared DTI values with the International Standards for Neurological Classification total motor and sensory scores ${ }^{5,6}$ and AIS (American Spinal Injury Association Impairment Scale) classification, ${ }^{4}$ our observation that FA values differ between subjects with SCI who have sacral sparing and those who do not have sacral sparing has not been previously reported. This finding has important clinical relevance as it is the sacral sparing end points that have received recent attention with respect to their validity and reliability and that are particularly difficult to evaluate ${ }^{16}$ in children. ${ }^{7,17}$ This study builds upon previous adult studies that show evidence in support of FA as a potential neuroimaging biomarker of the spinal cord and is the first to examine its diagnostic utility for sacral sparing end points in children.
As seen with FA, there were significant differences in diffusivity values between controls and subjects with complete SCI. However, diffusivity values did not differ between controls and subjects with incomplete injuries with the exception for subjects with anal contraction. The explanation for the difference in $\mathrm{AD}$ values between controls and subjects with intact anal contraction is not readily evident but may be due to the small sample size $(n=3)$ of subjects with anal contraction. As studies continue to establish DTI, a larger number of subjects in each AIS category will be needed to determine the utility of DTI for distinguishing among subgroups of pediatric SCI. Diffusivity has been less consistent as compared with FA when correlated with measures of impairment. ${ }^{5,12}$ A reason for this may be due to the characteristics of the samples studied. In this study, we found significant differences between the controls and the sample with complete injuries but not between controls and subjects with incomplete injuries.

Facon et al. ${ }^{18}$ reported that FA values differed between controls and patients at the MRI level of injury, but FA and AD values did not differ between controls and patients away from the MRI-defined injury site; they concluded that FA was better than $\mathrm{AD}$ and MRI for detecting acute and progressive SCI. Cheran et al. ${ }^{5}$ found differences in DTI values among controls, at the injury site and remote from the injury site. Like Facon et al., Cheran et al. studied patients acutely using a $1.5 \mathrm{~T}$ scanner. Peterson et al. ${ }^{4}$ utilized a $3 \mathrm{~T}$ scanner with subjects with subacute and chronic injuries. His findings were similar to the ones in this study in which FA and diffusivity values were different at the injury site as compared with cervical regions above and below the injury site. One possible explanation for differences among the various reports is errors in measured DTI values observed across MRI systems. Huisman et al. ${ }^{19}$ suggested that clinical studies using FA and diffusivity values should consequently compare them to normative FA and diffusivity value measurements that have been established for the field strength used. Thus, care must be taken when comparing results across reports, especially with regards to scanner field strength.

The strength of DTI as an imaging biomarker for areas above and below the injury level is important and requires further quantification. Unlike some of the reports on adults, in this study we scanned only the cervical cord (C1-T1). In future, we plan to scan the entire pediatric spinal cord. If DTI is able to quantify changes in the spinal cord at levels remote to the injury site, it would help fill a current void in practice and research by possibly quantifying the presumed changes that occur in the spinal cord due to restorative therapies such as body-weighted-supported treadmill training and functional electrical stimulation-assisted cycling. Currently, these rehabilitation paradigms are evaluated by secondary end points, such as the International Standards for Neurological Classification of Spinal Cord Injury. An imaging biomarker, such as DTI, may provide a quantitative method to evaluate the primary end point, changes at the spinal cord level. This capability would provide a potential additional benefit to evaluate pediatric SCI in which an estimated $10 \%$ of children who sustain traumatic cervical injuries sustain a second non-continuous SCI that is distal to the cervical injury and often unrecognized during the initial evaluation..$^{20}$ An imaging study using DTI may help to determine which injury has the most impact on clinical expression.

In this study, sensitivity and specificity values of DTI were evaluated for sacral sparing end points defined by the International Standards for Neurological Classification of Spinal Cord Injury, specifically S4-5 sensation, deep anal pressure and anal contraction. An interesting finding was the relatively low specificity values of DTI for anal contraction and deep anal pressure, particularly when 
compared with specificity for S4-5 sensation. Specificity reflects the degree to which a test correctly identifies a negative finding when the condition being tested for is truly absent. Although specificity of DTI for S4-5 sensation was relatively high (0.81), specificity for anal contraction and deep anal pressure was low (0.74 and 0.53, respectively), yielding a 26 and $47 \%$ incidence of false positives, respectively. These low values may have resulted from our method of drawing ROIs covering the entire cross-section of the cervical cord to establish DTI values as opposed to ROIs isolating tract-specific quadrants. In future, we plan to conduct quadrant analysis, which may potentially provide a more accurate indication of motor and sensory function. Another factor may be the inherent weaknesses of the deep anal pressure and anal contraction tests when conducted with children. In previous studies, we have shown that scores from sensory testing of S4-5 had strong reliability, whereas reliability of repeated scores for deep anal pressure and anal contraction were lower. ${ }^{7,16}$ Specificity and sensitivity of a new measure are largely dependent upon comparison to a valid gold-standard instrument. Despite the widespread use, there has been little work on the validation of the tests for sacral sparing. Results of this study provide further rationale to better establish the validity of the deep anal pressure and anal contraction examinations and the reliability of the scores they generate.

The combination of all three DTI values (FA, AD and RD) was the strongest predictor of both motor level ( $\mathrm{ROC}$ AUC $=0.92 ; 95 \%$ $\mathrm{CI}=0.91,0.94$ ) and MRI level of injury (ROC $\mathrm{AUC}=0.92$; $95 \% \mathrm{CI}=0.90,0.94$ ) (Figures 3 and 4 ). In cases of SCI, where there are breaches in white matter, demyelination and axon damage, FA, $\mathrm{AD}$ and $\mathrm{RD}$ each contribute unique information about the cord and collectively are strong predictors of motor and MRI levels of injury.

Spinal cord DTI is more complicated compared with brain DTI. Nonetheless, the implementation of the reduced-FoV technique resulted in reductions in geometric distortions, and distinctive delineation of gray and white matter structures were observed in the majority of slices; our previous reports on validity of this technique showed strong reproducibility ${ }^{13}$ and moderate-to-good clinical correlation. ${ }^{11}$ However, there are limitations to this study. Work on further development of methods to overcome artifact in the pediatric cord in needed. Additionally, further work is warranted on how best to accurately place ROI, particularly in the severely atrophied cord. The sample is small and variation in injury characteristics is large. Age- and gender-matched sampling methods were not used, and age was not controlled for in the analysis. The sample was not followed prospectively, and hence, information about therapies received and improvement made is not available. Future work will involve additional corss-sectional studies of DTI on the thoracic pediatric cord as well as longitudinal studies that will evaluate changes in DTI in relationship to changes in clinical end points.

\section{DATA ARCHIVING}

There were no data to deposit.

\section{CONFLICT OF INTEREST}

The authors declare no conflict of interest.

\section{ACKNOWLEDGEMENTS}

Christina Calhoun, MSPT and Laure Rutter, BSN assisted with recruitment and data collection. The study was funded by the Shriners Hospitals for Children Research Grant 8956 (Mulcahey, PI, USA).

1 Théaudin M, Sailou G, Denier C, Adams D, Ducreux D. A correlation between fractional anisotrophy variations and clinical recovery in spinal cord infarctions. J Neuroimaging 2013; 23: 256-258.

2 Kamble RB, Venkataramana NK, Naik AL, Rao SV. Diffusion tensor imaging in spinal cord injury. Indian J Radiol Imaging 2011; 21: 221-224.

3 Lindberg PG, Feydy A, Sanchez K, Rannou F, Maier MA. Measures of spinal canal steneosis and relationship to spinal cord structure in patients with cervical spondylosis. J Neuroradiol 2011; 39: 236-242.

4 Petersen JA, Wilm BJ, vonMeyenburg J, Schubert M, Seifert B, Najafi Y et al. Chronic cervical spinal cord injury: DTMRI correlates with clinical and electrophysiological measures. J Neurotrauma 2011; 29: 1556-1566.

5 Cheran S, Shanmuganathan K, Zhuo J, Mirvis SE, Aarabi B, Alexander MT et al. Correlation of MR diffusion tensor imaging parameters with ASIA motor scores in hemorrhagic and nonhemorrhagic acute spinal cord injury. J Neurotrauma 2011; 28: 1881-1892.

6 Cohen-Adad J, El Mendili M-M, Lehericy S, Pradat P-F, Blancho S, Rossignol S et al. Demyelination and degeneration in the injured human spinal cord detected with diffusion and magnetization transfer MRI. Neurolmage 2011; 55: 1024-1033.

7 Mulcahey MJ, Vogel L, Betz R, Samdani A, Chafetz R, Gaughan J. The international standards for neurological classification of spinal cord injury: psychometric evaluation and guidelines for use with children and youth. Phys Med Rehabil 2011; 92: 1264-1269.

8 Calhoun C, Chafetz R, Mulcahey MJ. Discrepancy between severity obtained from examinations and classification conducted by experienced examiners and classifiers and documented severity in paediatric spinal cord injury. Dev Neurorehabil 2009; 12: 406-410.

9 Mulcahey MJ, Gaughan J, Betz RR. Agreement of ISCSCI scores at individual myotomes and dermatomes in youth with complete injuries. Spinal Cord 2009; 47: 56-61.

10 Krisa L, Gaughan J, Vogel L, Betz RR, Mulcahey MJ. Agreement of repeated motor and sensory scres at individual myotomes and dermatomes in young persons with spinal cord injury. Spinal Cord 2012; 51: 75-81.

11 Mulcahey MJ, Samdani AF, Gaughan JP, Barakat N, Faro S, Betz RR et al. Diffusion tensor imaging in pediatric spinal cord injury: preliminary examination of reliability and clinical correlation. Spine 2011; 37: E797-E803.

12 Mohamed F, Hunter L, Barakat N, Chia-Shang J, Sair H, Samdani A et al. Diffusion tensor imaging of the pediatric spinal cord at $1.5 \mathrm{~T}$ : preliminary results. Am J Neuroradiol 2011; 32: 339-345.

13 Barakat N, Mohamed F, Hunter L, Shah L, Faro S, Samdani A et al. Diffusion tensor imaging of the normal pediatric spinal cord using an inner-FoV EPI sequence. Am J Neuroradiol 2012; 33: 1127-1133.

14 Kirshblum SC, Waring W, Biering-Sorensen F, Burns SP, Johansen M, Schmidt-Read M et al. Reference for the 2011 revision of the International Standards for Neurological Classification of Spinal Cord Injury. J Spinal Cord Med 2011; 34: 547-554.

15 Finsterbusch J. Eddy-current compensated diffusion weighting with a single refocusing RF pulse. Magn Reson Med 2009; 61: 748-754.

16 Van Middendorp JJ, Horman AJ, Pouw MH, van de Meent $\mathrm{H}$. Is determination between complete and incomplete traumatic spinal cord injury clinically relevant? Validation of the ASIA sacral sparing criteria in a prospective cohort of 432 patients. Spinal Cord 2009; 47: 809-816.

17 Vogel L, Samdani A, Chafetz R, Gaughan J, Mulcahey MJ. Intra-rater reliability of the anorectal exam and classification of injury in children with spinal cord injury. Spinal Cord 2009; 47: 687-691.

18 Facon D, Ozanne A, Fillard P, Lepeintre JF, Tournoux-Facon C, Ducreux D. MR diffusion tensor imaging and fiber tracking in spinal cord compression. Am J Neuroradiol 2005; 26: $1587-1594$

19 Huisman TA, Loenneker T, Barta G, Bellemann ME, Hennig J, Fischer JE et al. Quantitative diffusion tensor MR imaging of the brain: field strength related variance of apparent diffusion coefficient (ADC) and fractional anisotropy (FA) scalars. Eur Radiol 2006; 16: 1651-1658.

20 Mortazavi MM, Mariwalla NR, Horn EM, Tubbs RS, Theodore N. Absence of MRI soft tissue abnormalities in severe spinal cord injury in children: case-based update. Childs Nerv Syst 2011; 27: 1369-1373. 